\title{
Explicit Representation of the Minimal Variance Portfolio in Markets driven by Lévy processes
}

\author{
Fred Espen Benth ${ }^{1}$, Giulia Di Nunno ${ }^{1,2}$, Arne Løkka ${ }^{1}$, \\ Bernt Øksendal ${ }^{1,3}$ and Frank Proske ${ }^{1}$
}

April 18th, 2002.

\begin{abstract}
In a market driven by a Lévy martingale, we consider a claim $\xi$. We study the problem of minimal variance hedging and we give an explicit formula for the minimal variance portfolio in terms of Malliavin derivatives. We discuss two types of stochastic (Malliavin) derivatives for $\xi$ : one based on the chaos expansion in terms of iterated integrals with respect to the power jump processes and one based on the chaos expansion in terms of iterated integrals with respect to the Wiener process and the Poisson random measure components. We study the relation between these two expansions, the corresponding two derivatives and the corresponding versions of the Clark-Haussmann-Ocone theorem.

Key words: incomplete markets, minimal variance hedging, Lévy processes, stochastic derivatives, chaos expansions, Clark-Haussmann-Ocone theorem.

AMS (2000) Classification: primary 91B28; secondary 60H07, 60H05.
\end{abstract}

\section{Introduction.}

Suppose we have a financial market where the bond and stock prices $S_{0}, S_{1}, \ldots, S_{K}$ are modeled as follows:

$$
\text { Bond price: } \quad S_{0}(t)=1, \quad 0 \leq t \leq T,
$$

$$
\text { Stock prices: } \quad S_{j}(t)=\eta_{j}(t), \quad j=1, \ldots, K,
$$

where $\eta_{j}, j=1, \ldots, K$, are independent Lévy martingales of the second order on a filtered probability space $\left(\Omega, \mathfrak{A},\left\{\mathfrak{A}_{t}\right\}_{0 \leq t \leq T}, P\right)$ (i.e. the processes $\eta_{j}$ are Lévy processes and also martingales with finite second moment). Alternatively, to get a more realistic price model, we could assume that the stock prices were given by

$$
d S_{j}(t)=\sigma_{j}(t, \omega) S_{j}\left(t^{-}\right) d \eta_{j}(t), \quad 0 \leq t \leq T, \quad j=1, \ldots, K .
$$

\footnotetext{
${ }^{1}$ Department of Mathematics, University of Oslo, P.O. Box 1053 Blindern, N-0316 Oslo, Norway.

${ }^{2}$ Dipartimento di Matematica, Università degli Studi di Pavia, via Ferrata, 1, 27100 Pavia, Italy.

${ }^{3}$ Norwegian School of Economics and Business Administration, Helleveien 30, N-5045 Bergen, Norway. E-mail addresses. F.E. Benth: fredb@math.uio.no; G. Di Nunno: giulian@math.uio.no; A. Løkka: alokka@math.uio.no; B. Øksendal: oksendal@math.uio.no; F. Proske: proske@math.uio.no

Acknowledgements. The second author would like to thank the Department of Mathematics of the University of Oslo for their support and their warm hospitality during her stay there.
} 
However, since this only would require minor changes in the computations, we prefer to work with the simpler model (1.2) throughout this paper.

Suppose we are only allowed to trade in the first $k+1$ securities $S_{0}, S_{1}, \ldots S_{k}$, where $k \leq K$. A random variable $\xi \in L_{2}\left(\Omega, \mathfrak{A}_{T}, P\right)$ (a claim) is called replicable (or hedgeable) in this market if there exists a (predictable) adapted process $\varphi(t)=\left(\varphi_{1}(t), \ldots, \varphi_{k}(t)\right), 0 \leq t \leq T$ (a portfolio) such that $\varphi$ is admissible, i.e.

$$
\sum_{j=1}^{k} E\left[\int_{0}^{T} \varphi_{j}^{2}(s) d s\right]<\infty
$$

and such that $\varphi$ replicates (or hedges) $\xi$, in the sense that

$$
\xi=E \xi+\sum_{j=1}^{k} \int_{0}^{T} \varphi_{j}(s) d \eta_{j}(s), \quad \text { P-a.e. }
$$

where $E$ denotes the expectation with respect to $P$. In this case $\varphi(t), 0 \leq t \leq T$, is called hedging portfolio. The market is called complete if every $\xi \in L_{2}\left(\Omega, \mathfrak{A}_{T}, P\right)$ is replicable (hedgeable). The set of all admissible portfolios is denoted by $\mathcal{A}$.

It is well known that a market driven by a Lévy martingale is in general not complete. In view of this it is natural to ask: given a general Lévy martingale market as above and given $\xi \in L_{2}\left(\Omega, \mathfrak{A}_{T}, P\right)$, how close can we get to $\xi$ by hedging with an admissible portfolio $\varphi$ ? If we interpret closeness in terms of variance, the problem is to find $\varphi \in \mathcal{A}$ such that

$$
E\left[\left(\xi-E \xi-\sum_{j=1}^{k} \int_{0}^{T} \varphi_{j}(s) d \eta_{j}(s)\right)^{2}\right]=\inf _{\psi \in \mathcal{A}} E\left[\left(\xi-E \xi-\sum_{j=1}^{k} \int_{0}^{T} \psi_{j}(s) d \eta_{j}(s)\right)^{2}\right] .
$$

This question is often referred to the problem of minimal variance hedging for incomplete markets in mathematical finance. Here, we are assuming a market modeled by the above type Lévy process $\eta=\left(\eta_{1}, \ldots, \eta_{K}\right)$ and we assume that we are allowed to trade only in $\left(\eta_{1}, \ldots, \eta_{k}\right), k \leq K$. Thus the market can be incomplete for two reasons: the first is due to the random jumps of prices modeled by the Lévy process and the second is due to the trading constraints, $k \leq K$. A portfolio $\varphi$ that realizes the minimum is called a minimal variance portfolio.

This problem has been studied in the general context of martingales and semimartingales by many authors. See e.g. Chan (1999), Di Nunno (2001), Föllmer and Schweizer (1991), Föllmer and Sonderman (1986), Schweizer (2001), and the references therein.

The main contribution of this paper compared to the above papers is that, by specifying our study to Lévy martingales, we are able to give an explicit formula for the minimal variance portfolio $\varphi=\left(\varphi_{1}, \ldots, \varphi_{k}\right)$ in terms of the stochastic derivatives $D$ of the claim $\xi$. More precisely, we prove that

$$
\varphi_{j}(s)=\frac{1}{\mu_{j}}\left[\sigma_{j} E\left[D_{s, j} \xi \mid \mathfrak{A}_{s}\right]+\int_{\mathbb{R}} x E\left[D_{s, x, j} \xi \mid \mathfrak{A}_{s}\right] \nu_{j}(d x)\right], \quad j=1, \ldots, k .
$$

See Theorem 4.1. Here $\mu_{j}=\sigma_{j}^{2}+\int_{\mathbb{R}} x^{2} \nu_{j}(d x), \nu_{j}$ being the Lévy measure of $\eta_{j}$ and $\sigma_{j}$ the coefficient of the continuous (Wiener process) component $W_{j}$ of $\eta_{j}$, see Section 2. The 
symbols $D_{s, j}$ and $D_{s, x, j}$ denote the stochastic (Malliavin) derivatives in the directions of $W_{j}$ and $\tilde{N}_{j}$, where $\tilde{N}_{j}$ is the compensated Poisson random measure representing the pure jump component of $\eta_{j}$.

This paper is organized as follows. In Section 2 we recall some basic results about Lévy martingales and we relate our problem (1.5), to the the more general problem and its solution studied in Di Nunno (2001). In Section 3 we give a short review of chaos expansions, stochastic derivatives and Clark-Haussmann-Ocone theorems for Lévy processes, mainly based on Nualart and Schoutens (2000), Léon, Solé, Utzet and Vives (2002) and Løkka (2001). In Section 4 we prove our main results on minimal variance hedging and hedgeable claims. Finally, in Section 5, we illustrate our results by giving some explicit examples.

\section{Minimal variance portfolio and the general stochastic derivative $\mathfrak{D}$.}

For the standard $L_{2}$-space $H=L_{2}(\Omega)=L_{2}(\Omega, \mathfrak{A}, P)$ of real random variables $\xi$ :

$$
\|\xi\|=\left(\int_{\Omega}|\xi(\omega)|^{2} P(d \omega)\right)^{1 / 2}=\left(E|\xi|^{2}\right)^{1 / 2}
$$

on the filtered probability space $\left(\Omega, \mathfrak{A},\left\{\mathfrak{A}_{t}\right\}_{0 \leq t \leq T}, P\right)$, we consider the Itô type non-anticipating integration scheme with integrator as a $K$-dimensional Lévy process $\eta(t)=\left(\eta_{1}(t), \ldots, \eta_{K}(t)\right)$, $0 \leq t \leq T$, with independent $H$-valued components. Recall that a Lévy process is a stochastically continuous process with independent, stationary increments and $\eta(0)=0$ - cf. Protter (1990). Here we assume we are dealing with the existing càdlàg modification of the process. For more information about Lévy processes we refer to Bertoin (1996), Protter (1990) and Sato (1999). In this paper we confine ourselves to the case of Lévy processes $\eta(t), 0 \leq t \leq T$, as $\mathfrak{A}_{t}$-martingales in $H$, i.e. $E \eta_{j}(t)=0$ and $E \eta_{j}^{2}(t)=\mu_{j} t, 0 \leq t \leq T$, with stationary independent increments

$$
\Delta \eta_{j}:=\eta_{j}(t+\Delta t)-\eta_{j}(t)
$$

for the intervals $\Delta=(t, t+\Delta t] \subseteq(0, T]$, for all $j=1, \ldots, K$. In short we will name such processes Lévy martingales of the second order. Note that $\eta_{j}(t), 0 \leq t \leq T$, is $H$-continuous, i.e. $\left\|\eta_{j}(t)-\eta_{j}(s)\right\| \rightarrow 0, s \rightarrow t$.

From now on we assume the completed filtration $\mathfrak{A}_{t}, 0 \leq t \leq T$, to be generated by the integrator itself $\mathfrak{A}_{t}=\sigma\left\{\eta_{j}(s), 0 \leq s \leq t, j=1, \ldots, K\right\}$ and $\mathfrak{A}=\mathfrak{A}_{T}$. Note that this filtration is continuous.

For the considered type of Lévy processes $\eta_{t}, 0 \leq t \leq T$, we recall the following representation formula

$$
\eta_{j}(t)=\sigma_{j} W_{j}(t)+\int_{0}^{t} \int_{\mathbb{R}} x \tilde{N}_{j}(d s, d x), \quad 0 \leq t \leq T \quad(j=1, \ldots, K),
$$

where $W_{j}(t)$ is the standard Wiener process (with diffusion coefficient 1 ), $\sigma_{j}>0$ is a constant and $\tilde{N}_{j}(d t, d x)$ is the compensated Poisson random measure, i.e. $\tilde{N}_{j}(d t, d x)=N_{j}(d t, d x)-$ $\nu_{j}(d x) d t$ with $\nu_{j}(d x)$ as the jump measure (with finite second moment) of the Lévy process $\eta_{j}$ itself and $N_{j}(d t, d x)$ as a Poisson random measure such that $E N_{j}(d t, d x)=\nu_{j}(d x) d t$. 
For a given $\xi$ in $H$ we recall the following representation

$$
\xi=E \xi+\sum_{j=1}^{K} \int_{0}^{T} \theta_{j}(s) \sigma_{j} d W_{j}(s)+\sum_{j=1}^{K} \int_{0}^{T} \int_{\mathbb{R}} \Psi_{j}(s, x) \tilde{N}_{j}(d s, d x)
$$

with $\theta_{j}$ and $\Psi_{j}$ as well defined integrands for the corresponding integrals - cf. Ito (1956), for example. For the sake of completeness we remind the reader that $\theta_{j}(s), 0 \leq s \leq T$, and $\Psi_{j}(s, x), 0 \leq s \leq T, x \in \mathbb{R}$, are (predictable) adapted processes with $E\left[\int_{0}^{T} \int_{\mathbb{R}}\left|\theta_{j}(s)\right|^{2} d s\right]$ $<\infty$ and $E\left[\int_{0}^{T} \int_{\mathbb{R}}\left|\Psi_{j}(s, x)\right|^{2} \nu_{j}(d x) d s\right]<\infty$. We also recall the following Itô isometry:

$$
E \xi^{2}=(E \xi)^{2}+\sum_{j=1}^{K} \int_{0}^{T} E\left[\theta_{j}^{2}(s)\right] \sigma_{j}^{2} d s+\sum_{j=1}^{K} \int_{0}^{T} \int_{\mathbb{R}} E\left[\Psi_{j}^{2}(s, x)\right] \nu_{j}(d x) d s .
$$

Which claims $\xi \in H$ are the replicable ones? Using (2.2) we can give the following answer.

Theorem 2.1. The claim $\xi$ in $H$ such that

$$
\xi=E \xi+\sum_{j=1}^{K} \int_{0}^{T} \Phi_{j}(s) d W_{j}(s)+\sum_{j=1}^{K} \int_{0}^{T} \int_{\mathbb{R}} \Psi_{j}(s, x) \tilde{N}_{j}(d s, d x)
$$

is hedgeable if and only if the integrands have the following structure

$$
\begin{gathered}
\Phi_{j}(s)=\sigma_{j} \varphi_{j}(s) \quad \text { and } \quad \Psi_{j}(s, x)=\varphi_{j}(s) x, \quad \text { for } \quad 1 \leq j \leq k, \\
\text { and } \quad \Phi_{j}=\Psi_{j}=0, \quad \text { for } \quad k<j \leq K,
\end{gathered}
$$

with $0 \leq s \leq T$ and $x \in \mathbb{R}$, for some process $\varphi=\left(\varphi_{1}, \ldots, \varphi_{k}\right) \in \mathcal{A}$. If this is the case, then $\varphi=\left(\varphi_{1}, \ldots, \varphi_{k}\right)$ is the hedging strategy for $\xi$, i.e.

$$
\xi=E \xi+\sum_{j=1}^{k} \int_{0}^{T} \varphi_{j}(s) d \eta_{j}(s)
$$

Proof. For a hedgeable $\xi$ in $H$, from (2.4) and (2.6) we obtain

$$
\sum_{j=1}^{k} \int_{0}^{T} \varphi_{j}(s) d \eta_{j}(s)=\sum_{j=1}^{K}\left(\int_{0}^{T} \Phi_{j}(s) d W_{j}(s)+\int_{0}^{T} \int_{\mathbb{R}} \Psi_{j}(s, x) \tilde{N}_{j}(d s, d x)\right)
$$

and substituting the stochastic differential of $\eta_{t}$ - cf. (2.1), we have

$$
\begin{gathered}
\sum_{j=1}^{k} \int_{0}^{T}\left(\frac{1}{\sigma_{j}} \Phi_{j}(s)-\varphi_{j}(s)\right) \sigma_{j} d W_{j}(s)+\sum_{j=1}^{k} \int_{0}^{T} \int_{\mathbb{R}}\left(\Psi_{j}(s, x)-x \varphi_{j}(s)\right) \tilde{N}_{j}(d s, d x) \\
+\sum_{j=k+1}^{K}\left(\int_{0}^{T} \Phi_{j}(s) d W_{j}(s)+\int_{0}^{T} \int_{\mathbb{R}} \Psi_{j}(s, x) \tilde{N}_{j}(d s, d x)\right)=0 .
\end{gathered}
$$


Correspondingly we have

$$
\begin{gathered}
\sum_{j=1}^{k}\left(\int_{0}^{T} E\left[\left(\frac{1}{\sigma_{j}} \Phi_{j}(s)-\varphi_{j}(s)\right)^{2}\right] \sigma_{j}^{2} d s+\int_{0}^{T} \int_{\mathbb{R}} E\left[\left(\Psi_{j}(s, x)-x \varphi_{j}(s)\right)^{2}\right] \nu_{j}(d x) d s\right) \\
+\sum_{j=k+1}^{K}\left(\int_{0}^{T} E\left[\Phi_{j}^{2}(s)\right] d s+\int_{0}^{T} \int_{\mathbb{R}} E\left[\Psi_{j}^{2}(s, x)\right] \nu_{j}(d x) d s\right)=0 .
\end{gathered}
$$

Hence, (2.5) holds true. Conversely, suppose (2.5) holds. Then representation (2.4) becomes as follows

$$
\begin{gathered}
\xi=E \xi+\sum_{j=1}^{k}\left(\int_{0}^{T} \varphi_{j}(s) \sigma_{j} d W_{j}(s)+\int_{0}^{T} \int_{\mathbb{R}} x \varphi_{j}(s) \tilde{N}_{j}(d s, d x)\right) \\
=E \xi+\sum_{j=1}^{k} \int_{0}^{T} \varphi_{j}(s)\left(\sigma_{j} d W_{j}(s)+\int_{\mathbb{R}} x \tilde{N}_{j}(d s, d x)\right)=E \xi+\sum_{j=1}^{k} \int_{0}^{T} \varphi_{j}(s) d \eta_{j}(s) .
\end{gathered}
$$

Therefore $\xi$ is hedgeable. This ends the proof.

Let $L_{2}(\Omega \times[0, T])=L_{2}(\Omega \times[0, T], \mathfrak{A} \times \mathfrak{B}, P(d \omega) \times \lambda(d t))$, with $\mathfrak{B}$ the Borel $\sigma$-algebra of $[0, T]$ and $\lambda(d t)=d t$ the Lebesgue measure on $[0, T]$, be the standard functional $L_{2}$-space of random processes $\psi$ with the norm given by

$$
\|\psi\|_{L_{2}}=\left(\iint_{\Omega \times[0, T]}|\psi(\omega, t)|^{2} P(d \omega) \times d t\right)^{1 / 2}=\left(E \int_{0}^{T}|\psi(t)|^{2} d t\right)^{1 / 2}=\left(\int_{0}^{T} E|\psi(t)|^{2} d t\right)^{1 / 2} .
$$

The integrands $\varphi=\left(\varphi_{1}, \ldots, \varphi_{K}\right)$ are considered as elements of $\left(L_{2}(\Omega \times[0, T])\right)^{K}$. Here the integrands can be characterized as adapted processes, i.e. $\varphi(t)$ is $\mathfrak{A}_{t}$-measurable for all $t$, or equivalently, thanks to the direct product measure $P(d \omega) \times d t$ applied, as predictable processes, i.e. processes that are measurable with respect to the so-called predictable $\sigma$ algebra. Actually, integrands can be identified as limits

$$
\varphi=\lim _{h \rightarrow 0} \varphi^{h}, \quad \text { i.e. } \quad\left\|\varphi_{j}-\varphi_{j}^{h}\right\|_{L_{2}} \longrightarrow 0, \quad h \rightarrow 0, \quad j=1, \ldots, K,
$$

of simple functions $\varphi_{j}^{h}$ of the following form

$$
\varphi_{j}^{h}(s)=\sum_{\Delta} \varphi_{j, \Delta}^{h} 1_{\Delta}(s), \quad 0 \leq s \leq T,
$$

with $\varphi_{j, \Delta}^{h}$ as $\mathfrak{A}_{t}$-measurable elements in $H$ to the corresponding intervals $\Delta=(t, t+\Delta t]$ of the finite h-partition of $(0, T]: \sum \Delta=(0, T], \Delta t \leq h$.

In this framework, we are considering the problem of minimal variance hedging - cf. (1.5). In Di Nunno (2001) for the more general setup of $H$-continuous martingales with respect to a general filtration as integrators, an answer to the above question is suggested in terms of a certain general stochastic derivative. See also Di Nunno and Rozanov (1999). Here we recall its definition specifying it to the case of the Lévy martingales we are dealing with. 
Definition 2.2. For the random variable $\xi$ in $H$, the (non-anticipating) stochastic derivative

$$
\mathfrak{D}_{\eta_{j}} \xi:=\varphi_{j}=\varphi_{j}(s), \quad 0 \leq s \leq T,
$$

with respect to $\eta_{j}(t), 0 \leq t \leq T$, is an element of the functional space $L_{2}(\Omega \times[0, T])$ defined as the limit in (2.7) where the (2.8)-type simple functions are of the form

$$
\varphi_{j}^{h}(s)=\sum_{\Delta} E\left[\xi \cdot \frac{\Delta \eta_{j}}{\mu_{j} \Delta t} \mid \mathfrak{A}_{t}\right] 1_{\Delta}(s), \quad 0 \leq s \leq T .
$$

The stochastic function $\mathfrak{D}_{\eta} \xi=\left(\mathfrak{D}_{\eta_{1}} \xi, \ldots, \mathfrak{D}_{\eta_{K}} \xi\right)$ is referred to as the stochastic derivative with respect to the integrator $\eta=\left(\eta_{1}, \ldots, \eta_{K}\right)$. The stochastic derivative in Definition 2.2 is proved to be well defined for all $\xi \in H$. See Di Nunno (2001).

Let $H^{0} \subseteq H$ be the subspace of all variables $\xi$ characterized by $\mathfrak{D}_{\eta_{j}} \xi=0, j=1, \ldots, k$, and $H(\eta) \subseteq H$ the subspace of all stochastic integrals with integrator $\eta$. We can now formulate a theorem by Di Nunno (2001) on integral representation for random variables in $H$.

Theorem 2.3. Any $\xi$ in $H$ admits a unique integral representation in terms of its derivative $\mathfrak{D}_{\eta} \xi:$

$$
\xi=\xi^{0}+\sum_{j=1}^{k} \int_{0}^{T} \mathfrak{D}_{\eta_{j}} \xi(s) d \eta_{j}(s)
$$

with $\xi^{0} \in H^{0}$. Moreover, we have the following orthogonal decomposition:

$$
H=H^{0} \oplus H(\eta) .
$$

Note that the non-anticipating stochastic derivative $\mathfrak{D}_{\eta} \xi$ represents the minimal variance portfolio and the simple functions in Definition 2.2 give a method for the approximation.

The main purpose of this paper is to provide a more explicit formula for the integrand $\varphi=\mathfrak{D}_{\eta} \xi$ when $\eta_{j}, j=1, \ldots, K$, are independent Lévy martingales. For example, if $\eta=\left(\eta_{1}, \ldots, \eta_{K}\right)=\left(W_{1}, \ldots, W_{K}\right)$ is the Wiener process with independent components, the representation $(2.9)$, for an appropriate $\xi$, reduces to

$$
\xi=\xi^{0}+\sum_{j=1}^{k} \int_{0}^{T} E\left[D_{s, j} \xi \mid \mathfrak{A}_{s}\right] d W_{j}(s) .
$$

In other words in this case we have $\mathfrak{D}_{W_{j}} \xi(s)=E\left[D_{s, j} \xi \mid \mathfrak{A}_{s}\right], 0 \leq s \leq T$, where $D_{s, j} \xi$, $0 \leq s \leq T$, is the stochastic Malliavin derivative in the direction of the Wiener process $W_{j}$, cf. Malliavin (1997), for example. In particular, if $k=K$ this gives the well-known ClarkHaussmann-Ocone formula - cf. Nualart (1995), Øksendal (1996), Aase, Øksendal, Privault and Ubøe (2000), Karatzas, Ocone and Li (1991), for example.

\section{Chaos expansions and the stochastic derivatives $\mathcal{D}_{t}^{(m)}$ and $D_{t, x}$.}

In this section we review basic terminology and results about chaos expansions with respect to our Lévy martingales $\eta_{j}, j=1, \ldots, K$. For simplicity we assume that $K=1$ in this section and we write 


$$
\eta_{1}(t)=\eta(t)=\sigma W(t)+\int_{0}^{t} \int_{\mathbb{R}} x \tilde{N}(d s, d x)
$$

3.A. Chaos expansions in terms of iterated integrals with respect to power jump processes.

We first recall the chaos expansion of Nualart and Schoutens (2000), see also Léon, Solé, Utzet and Vives (2002) and Nualart and Schoutens (2001). Here we assume that for all $\varepsilon>0$ there exists $\delta>0$ such that

$$
\int_{\mathbb{R}-(-\varepsilon, \varepsilon)} \exp (\delta|x|) \nu(d x)<\infty
$$

Let $\Delta \eta(t)=\eta(t)-\eta\left(t^{-}\right)$be the jump of $\eta$ at time $t$ and let

$$
\eta^{(i)}(t)=\sum_{0<s \leq t}(\Delta \eta(s))^{i}, \quad 0 \leq t \leq T, \quad i=2,3, \ldots
$$

be the $i$-th power jump process, which is another Lévy process. Then

$$
E\left[\eta^{(i)}(t)\right]=t \int_{\mathbb{R}} x^{i} \nu(d x)=: m_{i} t, \quad i=2,3, \ldots
$$

We put $\eta^{(1)}(t):=\eta(t), 0 \leq t \leq T$, and $m_{1}:=0$. Hence we can define the compensated power jump process $\zeta^{(i)}(t), 0 \leq t \leq \bar{T}$, by

$$
\zeta^{(i)}(t):=\eta^{(i)}(t)-m_{i} t, \quad i=1,2, \ldots
$$

Finally, let $a_{i, j}$ be real numbers such that the processes

$$
\gamma^{(i)}(t):=\zeta^{(i)}(t)+a_{i, i-1} \zeta^{(i-1)}(t)+\cdots+a_{i, 1} \zeta^{(1)}(t)
$$

are strongly orthogonal martingales, in the sense that the quadratic covariation $\left[\gamma^{(i)}, \gamma^{(j)}\right]_{t}$, $0 \leq t \leq T$, is a uniformly integrable martingale for all $i \neq j, i, j=1,2, \ldots$ Then there are constants $M(i)>0$ such that $\left[\gamma^{(i)}, \gamma^{(i)}\right]_{t}=M(i) t$ - cf. Nualart and Schoutens (2000), Section 2. For all $n=1,2, \ldots$, let $\mathcal{I}_{n}$ denote the set of all multi-indices $\alpha=\left(\alpha_{1}, \ldots, \alpha_{n}\right)$ with $\alpha_{i} \in \mathbb{N}$ for $i=1, \ldots, n$ and put $\mathcal{I}=\cup_{n=1}^{\infty} \mathcal{I}_{n}$. For $\alpha=\left(\alpha_{1}, \ldots, \alpha_{n}\right) \in \mathcal{I}_{n}$, we write $l(\alpha)=n$ (length of $\alpha$ ) and we put

$$
S_{l(\alpha)}=S_{n}=\left\{\left(t_{1}, \ldots, t_{n}\right) \in[0, T]^{n}: 0 \leq t_{1} \leq \cdots \leq t_{n} \leq T\right\} .
$$

We also use the notation $d \gamma^{\otimes \alpha}(t)=d \gamma^{\left(\alpha_{1}\right)}\left(t_{1}\right) \ldots d \gamma^{\left(\alpha_{n}\right)}\left(t_{n}\right)$.

We can now formulate the theorem by Nualart and Schoutens (2000) on the chaos expansion of a random variable in terms of iterated integrals with respect to $\gamma^{(i)}$.

Theorem 3.1. (a) Every $\xi \in H$ can be written as follows

$$
\xi=E \xi+\sum_{n=1}^{\infty} \sum_{\alpha \in \mathcal{I}_{n}} \int_{0}^{T} \ldots \int_{0}^{t_{2}} f_{\alpha}\left(t_{1}, \ldots, t_{n}\right) d \gamma^{\left(\alpha_{1}\right)}\left(t_{1}\right) \ldots d \gamma^{\left(\alpha_{n}\right)}\left(t_{n}\right)
$$




$$
=E \xi+\sum_{\alpha \in \mathcal{I}} \int_{S_{l(\alpha)}} f_{\alpha}(\mathbf{t}) d \gamma^{\otimes \alpha}(\mathbf{t}),
$$

with $f_{\alpha} \in L_{2}\left(\mathbb{R}_{+}^{n}, \mathcal{B}\left(\mathbb{R}_{+}^{n}\right), \lambda^{n}\right)$.

(b) Moreover, we have the isometry

$$
\|\xi\|^{2}=E \xi^{2}=(E \xi)^{2}+\sum_{\alpha \in \mathcal{I}} M\left(\alpha_{1}\right) \ldots M\left(\alpha_{n}\right)\left\|f_{\alpha}\right\|_{S_{l(\alpha)}}^{2}
$$

where

$$
\left\|f_{\alpha}\right\|_{S_{l(\alpha)}}^{2}=\int_{0}^{T} \cdots \int_{0}^{t_{2}} f_{\alpha}^{2}\left(t_{1}, \ldots, t_{n}\right) d t_{1} \ldots d t_{n}=: \int_{S_{l(\alpha)}} f_{\alpha}^{2}(\mathbf{t}) d \mathbf{t}
$$

Corollary 3.2. Every $\xi \in H$ can be written as

$$
\xi=E \xi+\sum_{m=1}^{\infty} \int_{0}^{T} \varphi^{(m)}(s) d \gamma^{(m)}(s)
$$

where $\varphi^{(m)}, m=1,2, \ldots$, are predictable processes.

We now introduce a stochastic Sobolev space $\mathbf{D}_{1,2}$ and the stochastic derivative $\mathcal{D}_{t}^{(m)}$.

Definition 3.3. Let $\mathbf{D}_{1,2}$ denote the set of $\xi \in H$ the expansion (3.6) of which, satisfies the condition

$$
\|\xi\|_{\mathbf{D}_{1,2}}^{2}:=E \xi^{2}+\sum_{m=1}^{\infty} \sum_{n=1}^{\infty} \sum_{\substack{\alpha \in \mathcal{I}_{n} \\ \alpha_{n}=m}} M\left(\alpha_{1}\right) \ldots M\left(\alpha_{n-1}\right) \int_{0}^{T}\left\|f_{\alpha}(\cdot, t)\right\|_{S_{n-1}}^{2} d t<\infty .
$$

For $\xi \in \mathbf{D}_{1,2}$ we define its stochastic derivatives $\mathcal{D}_{t}^{(m)} \xi, 0 \leq t \leq T$, in $L_{2}(\Omega \times[0, T])$ for $m=1,2, \ldots$ by

$$
\mathcal{D}_{t}^{(m)} \xi=\sum_{n=1}^{\infty} \sum_{\substack{\alpha \in \mathcal{I}_{n} \\ \alpha_{n}=m}} \int_{S_{n-1}} f_{\alpha}\left(t_{1}, \ldots, t_{n-1}, t\right) d \gamma^{\left(\alpha_{1}\right)}\left(t_{1}\right) \ldots d \gamma^{\left(\alpha_{n-1}\right)}\left(t_{n-1}\right)
$$

where the summation is taken over all $\alpha=\left(\alpha_{1}, \ldots, \alpha_{n}\right) \in \mathcal{I}_{n}$ with the last non-zero element $\alpha_{n}=\alpha_{l(\alpha)}=m$.

Remark. This definition differs from the definition in Léon, Solé, Utzet and Vives (2002).

Note that if $\xi \in \mathbf{D}_{1,2}$ then $\mathcal{D}_{t}^{(m)} \xi \in L_{2}(\Omega \times[0, T])$ and

$$
\left\|\mathcal{D}_{t}^{(m)} \xi\right\|_{L_{2}(\Omega \times[0, T])}=\sum_{n=1}^{\infty} \sum_{\substack{\alpha \in \underline{\mathcal{I}}_{n} \\ \alpha_{n}=m}} M\left(\alpha_{1}\right) \ldots M\left(\alpha_{n-1}\right) \int_{0}^{T}\left\|f_{\alpha}(\cdot, t)\right\|_{S_{n-1}}^{2} d t<\infty .
$$

We can now give an explicit description of the integrands $\varphi^{(m)}$ in Corollary 3.2 in terms of the stochastic derivatives. This result corresponds to Theorem 1.6 in Léon, Solé, Utzet and Vives (2002).

Theorem 3.4. (Clark-Haussmann-Ocone theorem I) Let $\xi \in \mathbf{D}_{1,2}$. Then

$$
\xi=E \xi+\sum_{m=1}^{\infty} \int_{0}^{T} E\left[\mathcal{D}_{t}^{(m)} \xi \mid \mathfrak{A}_{t}\right] d \gamma^{(m)}(t) .
$$


Proof. Consider

$$
\begin{gathered}
\sum_{m=1}^{\infty} \int_{0}^{T} E\left[\mathcal{D}_{t}^{(m)} \xi \mid \mathfrak{A}_{t}\right] d \gamma^{(m)}(t) \\
=\sum_{m=1}^{\infty} \int_{0}^{T} E\left[\sum_{n=1}^{\infty} \sum_{\substack{\alpha \in \mathcal{I}_{n} \\
\alpha_{n}=m}} \int_{S_{n-1}} f_{\alpha}\left(t_{1}, \ldots, t_{n-1}, t\right) d \gamma^{\left(\alpha_{1}\right)}\left(t_{1}\right) \ldots d \gamma^{\left(\alpha_{n-1}\right)}\left(t_{n-1}\right) \mid \mathfrak{A}_{t}\right] d \gamma^{(m)}(t) \\
=\sum_{m=1}^{\infty} \sum_{n=1}^{\infty} \sum_{\substack{\alpha \in \mathcal{I}_{n} \\
\alpha_{n}=m}} \int_{0}^{T}\left(\int_{0 \leq t_{1} \leq \cdots \leq t_{n-1} \leq t} f_{\alpha}\left(t_{1}, \ldots, t_{n-1}, t\right) d \gamma^{\left(\alpha_{1}\right)}\left(t_{1}\right) \ldots d \gamma^{\left(\alpha_{n-1}\right)}\left(t_{n-1}\right)\right) d \gamma^{(m)}(t) \\
=\sum_{m=1}^{\infty} \sum_{n=1}^{\infty} \sum_{\substack{\alpha \in \mathcal{I}_{n} \\
\alpha_{n}=m}} \int_{S_{n}} f_{\alpha}(\mathbf{t}) d \gamma^{\otimes \alpha}(\mathbf{t})=\xi-E \xi
\end{gathered}
$$

by Theorem 3.1 .

\section{B. Chaos expansions in terms of iterated integrals with respect to Poisson random measures.}

Next we recall the chaos expansion presented in Løkka (2001). For simplicity in notation we assume, as in Løkka (2001), that $\sigma=0$, so that representation (2.1) reduces to

$$
\eta(t)=\int_{0}^{t} \int_{\mathbb{R}} x \tilde{N}(d s, d x), \quad 0 \leq t \leq T
$$

The chaos expansion is in terms of iterated integrals with respect to the compensated Poisson measure $\tilde{N}(d t, d x)=N(d t, d x)-\nu(d x) d t$. In the following we let $L_{2}\left((\lambda \times \nu)^{n}\right)$ be the space of deterministic functions $f:([0, T] \times \mathbb{R})^{n} \longrightarrow \mathbb{R}$ such that

$$
\|f\|_{L_{2}\left((\lambda \times \nu)^{n}\right)}^{2}:=\int_{([0, T] \times \mathbb{R})^{n}} f^{2}\left(t_{1}, x_{1}, \ldots, t_{n}, x_{n}\right) d t_{1} \nu\left(d x_{1}\right) \ldots d t_{n} \nu\left(d x_{n}\right)<\infty .
$$

Recall that $d \lambda(t)=d t$ denotes the Lebesgue measure on $[0, T]$. If $f$ is a real function on $([0, T] \times \mathbb{R})^{n}$ we define its symmetrization $\tilde{f}$ with respect to the variables $\left(t_{1}, x_{1}\right), \ldots,\left(t_{n}, x_{n}\right)$ by

$$
\tilde{f}\left(t_{1}, x_{1}, \ldots, t_{n}, x_{n}\right)=\frac{1}{n !} \sum_{\sigma} f\left(t_{\sigma_{1}}, x_{\sigma_{1}}, \ldots, t_{\sigma_{n}}, x_{\sigma_{n}}\right)
$$

where the sum is taken over all permutations $\sigma$ of $\{1, \ldots, n\}$. We say that $f$ is symmetric if $f=\tilde{f}$ and we let $\tilde{L}_{2}\left((\lambda \times \nu)^{n}\right)$ denote the set of symmetric deterministic functions in $L_{2}\left((\lambda \times \nu)^{n}\right)$. Moreover, we put

$$
G_{n}=\left\{\left(t_{1}, x_{1}, \ldots, t_{n}, x_{n}\right): 0 \leq t_{1} \leq \cdots \leq t_{n} \leq T ; x_{i} \in \mathbb{R}, i=1, \ldots, n\right\},
$$

and we denote by $L_{2}\left(G_{n}\right)$ the space of functions $g$ such that

$$
\|g\|_{L_{2}\left(G_{n}\right)}^{2}=\int_{G_{n}} g^{2}\left(t_{1}, x_{1}, \ldots, t_{n}, x_{n}\right) d t_{1} \nu\left(d x_{1}\right) \ldots d t_{n} \nu\left(d x_{n}\right)<\infty .
$$


Naturally, for $f \in \tilde{L}_{2}\left((\lambda \times \nu)^{n}\right)$ we have $\|f\|_{L_{2}\left((\lambda \times \nu)^{n}\right)}^{2}=n !\|f\|_{L_{2}\left(G_{n}\right)}^{2}$.

If $f \in L_{2}\left(G_{n}\right)$ we define its $n$-fold iterated integral by

$$
J_{n}(f):=\int_{0}^{T} \int_{\mathbb{R}} \ldots \int_{0}^{t_{2}} \int_{\mathbb{R}} f\left(t_{1}, x_{1}, \ldots, t_{n}, x_{n}\right) \tilde{N}\left(d t_{1}, d x_{1}\right) \ldots \tilde{N}\left(d t_{n}, d x_{n}\right)
$$

If $f \in \tilde{L}_{2}\left((\lambda \times \nu)^{n}\right)$ we define

$$
I_{n}(f)=\int_{([0, T] \times \mathbb{R})^{n}} f\left(t_{1}, x_{1}, \ldots, t_{n}, x_{n}\right) \tilde{N}^{\otimes n}(d \mathbf{t}, d \mathbf{x})=n ! J_{n}(f) .
$$

Then if $f \in \tilde{L}_{2}\left((\lambda \times \nu)^{n}\right)$ and $g \in \tilde{L}_{2}\left((\lambda \times \nu)^{m}\right)$ we have

$$
E\left[I_{n}(f) I_{m}(g)\right]= \begin{cases}0, & n \neq m \\ n !(f, g)_{L_{2}\left((\lambda \times \nu)^{n}\right)}, & n=m\end{cases}
$$

where

$$
(f, g)_{L_{2}\left((\lambda \times \nu)^{n}\right)}=\int_{([0, T] \times \mathbb{R})^{n}} f\left(t_{1}, x_{1}, \ldots, t_{n}, x_{n}\right) g\left(t_{1}, x_{1}, \ldots, t_{n}, x_{n}\right)(\lambda \times \nu)^{\otimes n}(d \mathbf{t}, d \mathbf{x}) .
$$

We can now formulate the theorem by Løkka (2001) on chaos expansion in terms of iterated integrals with respect to $\tilde{N}(d t, d x)$.

Theorem 3.5. (a) Every $\xi \in H$ can be written as

$$
\xi=E \xi+\sum_{n=1}^{\infty} I_{n}\left(f_{n}\right)
$$

for a unique sequence $\left\{f_{n}\right\}_{n=1}^{\infty}$ with $f_{n} \in \tilde{L}_{2}\left((\lambda \times \nu)^{n}\right)$.

(b) Moreover, we have the following isometry

$$
E \xi^{2}=(E \xi)^{2}+\sum_{n=1}^{\infty} n !\left\|f_{n}\right\|_{L_{2}\left((\lambda \times \nu)^{n}\right)}^{2} .
$$

Based on this expansion we define another stochastic Sobolev space $\mathbb{D}_{1,2}$ and the stochastic derivative $D_{t, x}$ as follows.

Definition 3.6. (a) We define $\mathbb{D}_{1,2}$ to be the set of $\xi \in H$ the expansion (3.15) of which satisfies the condition

$$
\|\xi\|_{\mathbb{D}_{1,2}}^{2}:=E \xi^{2}+\sum_{n=1}^{\infty} n n !\left\|f_{n}\right\|_{L_{2}\left((\lambda \times \nu)^{n}\right)}^{2}<\infty .
$$

(b) If $\xi \in \mathbb{D}_{1,2}$ we define its stochastic derivative $D_{t, x} \xi \in L_{2}(\Omega \times[0, T] \times \mathbb{R})$ by

$$
D_{t, x} \xi=\sum_{n=1}^{\infty} n I_{n-1}\left(f_{n}(\cdot, t, x)\right)
$$


where $f_{n}(\cdot, t, x)=f_{n}\left(t_{1}, x_{1}, \ldots, t_{n-1}, x_{n-1}, t, x\right)$.

The following isometry is obtained in Løkka (2001):

$$
\left\|D_{t, x} \xi\right\|_{L_{2}(\Omega \times[0, T] \times \mathbb{R})}^{2}=\sum_{n=1}^{\infty} n n !\left\|f_{n}\right\|_{L_{2}\left((\lambda \times \nu)^{n}\right)}^{2} .
$$

Corresponding to Theorem 3.4 we now have

Theorem 3.7. (Clark-Haussmann-Ocone theorem II). Let $\xi \in \mathbb{D}_{1,2}$. Then

$$
\xi=E \xi+\int_{0}^{T} \int_{\mathbb{R}} E\left[D_{t, x} \xi \mid \mathfrak{A}_{t}\right] \tilde{N}(d t, d x) .
$$

\section{C. Relations between the two chaos expansions and the two stochastic deriva- tives.}

For the sake of completeness we point out the links between the chaos expansions and the stochastic derivatives presented so far in this section. Recall that here we are dealing with a pure jump Lévy martingale satisfying condition (3.2).

First of all we see that the compensated power jump process in (3.4) satisfies the equation

$$
\zeta^{(i)}(t)=\int_{0}^{t} \int_{\mathbb{R}} x^{i} \tilde{N}(d s, d x), \quad 0 \leq t \leq T, \quad i=1,2, \ldots,
$$

and therefore from (3.5) we get

$$
\gamma^{(i)}(t)=\sum_{j=1}^{i} a_{i, j} \zeta^{(j)}(t)=\sum_{j=1}^{i} a_{i, j} \int_{0}^{t} \int_{\mathbb{R}} x^{j} \tilde{N}(d s, d x), \quad 0 \leq t \leq T,
$$

with $a_{i, i}=1$, for all $i=1,2, \ldots$.

By the following equalities we show how the chaos expansions are related. From Theorem 3.1 , for any $\xi \in H$, we have

$$
\begin{gathered}
\xi-E \xi=\sum_{n=1}^{\infty} \sum_{\alpha \in \mathcal{I}_{n}} \int_{0}^{T} \ldots \int_{0}^{t_{2}} f_{\alpha}\left(t_{1}, \ldots, t_{n}\right) d \gamma^{\left(\alpha_{1}\right)}\left(t_{1}\right) \ldots d \gamma^{\left(\alpha_{n}\right)}\left(t_{n}\right) \\
=\sum_{n=1}^{\infty} \sum_{\alpha \in \mathcal{I}_{n}} \int_{0}^{T} \int_{\mathbb{R}} \ldots \int_{0}^{t_{2}} \int_{\mathbb{R}} \sum_{j_{1}=1}^{\alpha_{1}} \ldots \sum_{j_{n}=1}^{\alpha_{n}} a_{\alpha_{1}, j_{1}} \ldots a_{\alpha_{n}, j_{n}} x_{1}^{j_{1}} \ldots x_{n}^{j_{n}} f_{\alpha}\left(t_{1}, \ldots, t_{n}\right) \tilde{N}\left(d t_{1}, d x_{1}\right) \ldots \tilde{N}\left(d t_{n}, d x_{n}\right) \\
=\sum_{n=1}^{\infty} \int_{0}^{T} \int_{\mathbb{R}} \ldots \int_{0}^{t_{2}} \int_{\mathbb{R}} g_{n}\left(t_{1}, x_{1}, \ldots, t_{n}, x_{n}\right) \tilde{N}\left(d t_{1}, d x_{1}\right) \ldots \tilde{N}\left(d t_{n}, d x_{n}\right) \\
=\sum_{n=1}^{\infty} J_{n}\left(g_{n}\right)=\sum_{n=1}^{\infty} n ! J_{n}\left(\tilde{g}_{n}\right)=\sum_{n=1}^{\infty} I_{n}\left(\tilde{g}_{n}\right),
\end{gathered}
$$

where $\tilde{g}_{n}$ is the symmetrization (in the sense of (3.12)) of the function $g_{n}$ defined by

$$
g_{n}\left(t_{1}, x_{1}, \ldots, t_{n}, x_{n}\right)=
$$




$$
\begin{cases}\sum_{\alpha \in \mathcal{I}_{n}} \sum_{j_{1}=1}^{\alpha_{1}} \cdots \sum_{j_{n}=1}^{\alpha_{n}} a_{\alpha_{1}, j_{1}} \ldots a_{\alpha_{n}, j_{n}} x_{1}^{j_{1}} \ldots x_{n}^{j_{n}} f_{\alpha}\left(t_{1}, \ldots, t_{n}\right), & \text { on } G_{n} \\ 0, & \text { on }([0, T] \times \mathbb{R})^{n}-G_{n} .\end{cases}
$$

Therefore, by uniqueness, $\left\{f_{n}\right\}_{n=1}^{\infty}$ in (3.15) is given by

$$
f_{n}=\tilde{g}_{n}, \quad n=1,2, \ldots
$$

This gives the relation between the two expansions.

The relationship between the stochastic derivatives now comes immediately. In fact, from Definition 3.6 we have

$$
\begin{gathered}
E\left[D_{t, x} \xi \mid \mathfrak{A}_{t}\right]=\sum_{n=1}^{\infty} n E\left[I_{n-1}\left(\tilde{g}_{n}(\cdot, t, x)\right) \mid \mathfrak{A}_{t}\right]=\sum_{n=1}^{\infty} n(n-1) ! E\left[J_{n-1}\left(\tilde{g}_{n}(\cdot, t, x)\right) \mid \mathfrak{A}_{t}\right] \\
=\sum_{n=1}^{\infty} \int_{0}^{t} \int_{\mathbb{R}} \ldots \int_{0}^{t_{2}} \int_{\mathbb{R}} g_{n}\left(t_{1}, x_{1}, \ldots, t_{n-1}, x_{n-1}, t, x\right) \tilde{N}\left(d t_{1}, d x_{1}\right) \ldots \tilde{N}\left(d t_{n-1}, d x_{n-1}\right) \\
=\sum_{n=1}^{\infty} \sum_{\alpha \in \mathcal{I}_{n}} \int_{0}^{t} \int_{\mathbb{R}} \ldots \int_{0}^{t_{2}} \int_{\mathbb{R}} \sum_{j_{1}=1}^{\alpha_{1}} \ldots \sum_{j_{n}=1}^{\alpha_{n}} a_{\alpha_{1}, j_{1}} \ldots a_{\alpha_{n}, j_{n}} x_{1}^{j_{1}} \ldots x_{n-1}^{j_{n-1}} x^{j_{n}} \\
f_{\alpha}\left(t_{1}, \ldots, t_{n-1}, t\right) \tilde{N}\left(d t_{1}, d x_{1}\right) \ldots \tilde{N}\left(d t_{n-1}, d x_{n-1}\right) .
\end{gathered}
$$

Now $d \gamma^{\left(\alpha_{i}\right)}(t)=\sum_{j=1}^{\alpha_{i}} a_{\alpha_{i}, j} d \zeta^{(j)}=\int_{\mathbb{R}} \sum_{j=1}^{\alpha_{i}} a_{\alpha_{i}, j} x^{j} \tilde{N}(d t, d x)$ and therefore we get from the above

$$
\begin{gathered}
E\left[D_{t, x} \xi \mid \mathfrak{A}_{t}\right]=\sum_{n=1}^{\infty} \sum_{\alpha \in \mathcal{I}_{n}} \sum_{j_{n}=1}^{\alpha_{n}}\left(\int_{0}^{t} \ldots \int_{0}^{t_{2}} f_{\alpha}\left(t_{1}, \ldots, t_{n-1}, t\right) d \gamma^{\left(\alpha_{1}\right)}\left(t_{1}\right) \ldots d \gamma^{\left(\alpha_{n-1}\right)}\left(t_{n-1}\right)\right) a_{\alpha_{n}, j_{n}} x^{j_{n}} \\
=\sum_{n=1}^{\infty} \sum_{m=1}^{\infty} \sum_{\substack{\alpha \in \mathcal{I}_{n} \\
\alpha_{n}=m}} \sum_{j=1}^{m} a_{m, j} x^{j}\left(\int_{0}^{t} \ldots \int_{0}^{t_{2}} f_{\alpha}\left(t_{1}, \ldots, t_{n-1}, t\right) d \gamma^{\left(\alpha_{1}\right)}\left(t_{1}\right) \ldots d \gamma^{\left(\alpha_{n-1}\right)}\left(t_{n-1}\right)\right) \\
=\sum_{m=1}^{\infty} \sum_{j=1}^{m} a_{m, j} x^{j} E\left[\mathcal{D}_{t}^{(m)} \xi \mid \mathfrak{A}_{t}\right] .
\end{gathered}
$$

Thus, we have proved the following

Proposition 3.8. The relation between the two expansions of Theorem 3.1 and Theorem 3.5 is given by (3.22) and (3.21). For any $\xi \in \mathbf{D}_{1,2} \cap \mathbb{D}_{1,2}$ the stochastic derivatives in Definition 3.3 and Definition 3.6 satisfy the following relation

$$
E\left[D_{t, x} \xi \mid \mathfrak{A}_{t}\right]=\sum_{m=1}^{\infty} \sum_{j=1}^{m} a_{m, j} x^{j} E\left[\mathcal{D}_{t}^{(m)} \xi \mid \mathfrak{A}_{t}\right]
$$

\section{D. The case with both a continuous (Wiener process) component and a pure jump (Poisson random measure) component.}

In the case of a general Lévy martingale - cf. (3.1), the chaos expansion and the stochastic (Malliavin) derivative for a certain $\xi$ in the corresponding Sobolev type space $\mathbb{D}_{1,2}$ can still 
be defined following the same lines as in Ito (1956) and Løkka (2001). But in this case the multiple integrals of the chaos expansion are expressed in terms of mixtures of both the continuous components and the pure jump components. For an explanation we require the following "unified" notation:

$$
\begin{aligned}
& U_{1}=[0, T] \quad \text { and } \quad U_{2}=[0, T] \times \mathbb{R} \\
& d Q_{1}(\cdot)=d W(\cdot) \quad \text { and } \quad Q_{2}(\cdot)=\tilde{N}(\cdot, \cdot) \\
& d\left\langle Q_{1}\right\rangle=d \lambda \quad \text { and } \quad d\left\langle Q_{2}\right\rangle=d \lambda \times d \nu \\
& \int_{U_{1}} g\left(u^{(1)}\right) Q_{1}\left(d u^{(1)}\right)=\int_{0}^{t} g(s) W(d s) \quad \text { and } \int_{U_{2}} g\left(u^{(2)}\right) Q_{2}\left(d u^{(2)}\right)=\int_{0}^{t} \int_{\mathbb{R}} g(s, x) \tilde{N}(d s, d x)
\end{aligned}
$$

Theorem 3.9. For any $\xi \in H$

$$
\xi=E \xi+\sum_{n=1}^{\infty} \sum_{j_{1}, \ldots, j_{n}=1,2} J_{n}\left(g_{n}^{\left(j_{1}, \ldots, j_{n}\right)}\right)
$$

for a unique sequence $g_{n}^{\left(j_{1}, \ldots, j_{n}\right)}\left(j_{1}, \ldots, j_{n}=1,2 ; n=1,2, \ldots\right)$, of deterministic functions in the corresponding $L_{2}$-space $\left.L_{2}\left(G_{n}\right)=L_{2}\left(G_{n}, \bigotimes_{i=1}^{n} d\left\langle Q_{j_{i}}\right\rangle\right)\right)$, where $G_{n}=\left\{\left(u_{1}^{\left(j_{1}\right)}, \ldots, u_{n}^{\left(j_{n}\right)}\right) \in\right.$ $\left.\prod_{i=1}^{n} U_{j_{i}}: 0 \leq t_{1} \leq \ldots \leq t_{n} \leq T\right\}$ with $u^{\left(j_{i}\right)}=t$, if $j_{i}=1$, and $u^{\left(j_{i}\right)}=(t, x)$, if $j_{i}=2$, and $J_{n}\left(g_{n}^{\left(j_{1}, \ldots, j_{n}\right)}\right)=\int_{\prod_{i=1}^{n} U_{j_{i}}} g_{n}^{\left(j_{1}, \ldots, j_{n}\right)}\left(u_{1}^{\left(j_{1}\right)}, \ldots, u_{n}^{\left(j_{n}\right)}\right) 1_{G_{n}}\left(u_{1}^{\left(j_{1}\right)}, \ldots, u_{n}^{\left(j_{n}\right)}\right) Q_{j_{1}}\left(d u_{1}^{\left(j_{1}\right)}\right) \ldots Q_{j_{n}}\left(d u_{n}^{\left(j_{n}\right)}\right)$.

Based on the above expansion the stochastic Sobolev space $\mathbb{D}_{1,2}$ and the stochastic derivative $D$ are as follows.

Definition 3.10. (a) $\mathbb{D}_{1,2}$ is the set of all $\xi \in H$ the expansion (3.24) of which satisfies the condition

$$
\|\xi\|_{\mathbb{D}_{1,2}}^{2}=E \xi^{2}+\sum_{n=1}^{\infty} \sum_{j_{1}, \ldots, j_{n}=1,2} \int_{U_{j_{n}}}\left\|g_{n}^{\left(j_{1}, \ldots, j_{n}\right)}\left(\cdot, u_{n}^{\left(j_{n}\right)}\right)\right\|_{L_{2}\left(G_{n-1}\right)}^{2} d\left\langle Q_{j_{n}}\right\rangle\left(u_{n}^{\left(j_{n}\right)}\right)<\infty .
$$

(b) If $\xi \in \mathbb{D}_{1,2}$, we define its stochastic derivative as follows:

$$
D_{u}^{\{i\}} \xi=\sum_{n=1}^{\infty} \sum_{j_{1}, \ldots, j_{n-1}=1,2} J_{n-1}\left(g_{n}^{\left(j_{1}, \ldots, j_{n-1}, i\right)}\left(\cdot, u^{(i)}\right)\right), \quad i=1,2
$$

i.e.

$$
\begin{aligned}
D_{t} \xi & =\sum_{n=1}^{\infty} \sum_{j_{1}, \ldots, j_{n-1}=1,2} J_{n-1}\left(g_{n}^{\left(j_{1}, \ldots, j_{n-1}, 1\right)}(\cdot, t)\right), \quad i=1, \\
D_{t, x} \xi & =\sum_{n=1}^{\infty} \sum_{j_{1}, \ldots, j_{n-1}=1,2} J_{n-1}\left(g_{n}^{\left(j_{1}, \ldots, j_{n-1}, 2\right)}(\cdot, t, x)\right), \quad i=2 .
\end{aligned}
$$

Note that if $\xi \in \mathbb{D}_{1,2}$ then

$$
\sum_{n=1}^{\infty} \sum_{j_{1}, \ldots, j_{n}=1,2} \int_{U_{j_{n}}}\left\|g_{n}^{\left(j_{1}, \ldots, j_{n}\right)}\left(\cdot, u_{n}^{\left(j_{n}\right)}\right)\right\|_{L_{2}\left(G_{n-1}\right)}^{2} d\left\langle Q_{j_{n}}\right\rangle\left(u_{n}^{\left(j_{n}\right)}\right)=\sum_{i=1,2} E\left[\int_{U_{i}}\left|D_{u}^{\{i\}} \xi\right|^{2} d\left\langle Q_{i}\right\rangle\left(u^{(i)}\right)\right] .
$$


It is worth mentioning here that, in the case of the pure jump Lévy martingale, it is possible to define a derivative operator $D_{t, x}$ on $\mathbb{D}_{1,2}$ by a certain difference operator. See $\mathrm{L}$ okka (2001).

To state the last result of this section we consider again the representation (2.1) in the multidimensional setup. The derivative of $\xi$ with respect to the Wiener process $W_{j}$ will be denoted by $D_{s, j} \xi, 0 \leq s \leq T$, and the derivative with respect to the $j$-th compensated Poisson random measure will be $D_{s, x, j} \xi, 0 \leq s \leq T, x \in \mathbb{R}$, here and further on in this paper.

Theorem 3.11. For any $\xi \in \mathbb{D}_{1,2}$, representation (2.2) can be specified as follows

$$
\xi=E \xi+\sum_{j=1}^{K} \int_{0}^{T} E\left[D_{s, j} \xi \mid \mathfrak{A}_{s}\right] d W_{j}(s)+\sum_{j=1}^{K} \int_{0}^{T} \int_{\mathbb{R}} E\left[D_{s, x, j} \xi \mid \mathfrak{A}_{s}\right] \tilde{N}_{j}(d s, d x) .
$$

Similarly we can state the multidimentional version of Corollary 3.2 and Theorem 3.4. Note that we use the corresponding multidimensional notation.

Theorem 3.12. (a) Any $\xi \in H$, can be written as

$$
\xi=E \xi+\sum_{j=1}^{K} \sum_{m=1}^{\infty} \int_{0}^{T} \varphi_{j}^{(m)}(s) d \gamma_{j}^{(m)}(s) .
$$

(b) Moreover any $\xi \in \mathbf{D}_{1,2}$ admits the following specification of (3.26):

$$
\xi=E \xi+\sum_{j=1}^{K} \sum_{m=1}^{\infty} \int_{0}^{T} E\left[\mathcal{D}_{s, j}^{(m)} \xi \mid \mathfrak{A}_{s}\right] d \gamma_{j}^{(m)}(s) .
$$

Here $\mathbf{D}_{1,2}$ is the space of all $\xi \in H$ such that

$$
\xi=E \xi+\sum_{j=1}^{K} \sum_{\alpha \in \mathcal{I}} \int_{S_{l(\alpha)}} f_{\alpha, j}(\mathbf{t}) d \gamma_{j}^{\otimes \alpha}(\mathbf{t}),
$$

satisfies

$$
\|\xi\|_{\mathbf{D}_{1,2}}^{2}:=E \xi^{2}+\sum_{j=1}^{K} \sum_{m=1}^{\infty} \sum_{n=1}^{\infty} \sum_{\substack{\alpha=\left(\alpha_{1}, \ldots, \alpha_{n}\right) \in \mathcal{I} \\ \alpha_{n}=m}} M_{j}\left(\alpha_{1}\right) \ldots M_{j}\left(\alpha_{n-1}\right) \int_{0}^{T}\left\|f_{\alpha, j}(\cdot, t)\right\|_{S_{n-1}}^{2} d t<\infty
$$

with $\left[\gamma_{j}^{(i)}, \gamma_{j}^{(i)}\right]_{t}=M_{j}(i) t, 0 \leq t \leq T, i=1,2, \ldots$

\section{Minimal variance portfolio and explicit representation formulae.}

In the line suggested by Di Nunno (2001) and exploiting representation (2.2) and its specification in Theorem 3.11, we can now give an explicit formula for the minimal variance portfolio. Our result may be regarded as a generalization of the result obtained in Lamberton and Lapeyre (1996), p. 155, for the sum of a Wiener process and a Poisson process.

Theorem 4.1. For any $\xi \in \mathbb{D}_{1,2}$, the minimal variance portfolio $\varphi=\left(\varphi_{1}, \ldots, \varphi_{k}\right)$ in (1.5): 


$$
\hat{\xi}=E \xi+\sum_{j=1}^{k} \int_{0}^{T} \varphi_{j}(s) d \eta_{j}(s)
$$

admits the following representation

$$
\varphi_{j}(s):=\mathfrak{D}_{\eta_{j}} \xi(s)=\frac{1}{\mu_{j}}\left[\sigma_{j} E\left[D_{s, j} \xi \mid \mathfrak{A}_{s}\right]+\int_{\mathbb{R}} x E\left[D_{s, x, j} \xi \mid \mathfrak{A}_{s}\right] \nu_{j}(d x)\right]
$$

where $\mu_{j}=\sigma_{j}^{2}+\int_{\mathbb{R}} x^{2} \nu_{j}(d x)$ in $E \eta_{j}^{2}(t)=\mu_{j} t, 0 \leq t \leq T$.

Proof. By the Hilbert space argument, $H=H^{0} \oplus H(\eta)$, in the line of Theorem 2.3, the following orthogonality condition is satisfied

$$
E[(\xi-\hat{\xi}) \Theta]=E\left[\xi^{0} \Theta\right]=0, \quad \forall \Theta \in H(\eta),
$$

where

$$
\Theta=\sum_{j=1}^{k} \int_{0}^{T} \theta_{j}(s) d \eta_{j}(s)=\sum_{j=1}^{k} \int_{0}^{T} \theta_{j}(s) \sigma_{j} d W_{j}(s)+\sum_{j=1}^{k} \int_{0}^{T} \int_{\mathbb{R}} x \theta_{j}(s) \tilde{N}_{j}(d x, d s)
$$

for all $\theta=\left(\theta_{1}, \ldots, \theta_{k}\right) \in \mathcal{A}$, admissible portfolios, and $\xi^{0}=\xi-\sum_{j=1}^{k} \int_{0}^{T} \mathfrak{D}_{\eta_{j}} \xi(s) d \eta_{j}(s)$. From Theorem 3.11 we can write

$\xi^{0}=E \xi+\sum_{j=1}^{k} \int_{0}^{T}\left(\frac{1}{\sigma_{j}} E\left[D_{s, j} \xi \mid \mathfrak{A}_{s}\right]-\mathfrak{D}_{\eta_{j}} \xi(s)\right) \sigma_{j} d W_{j}(s)+\sum_{j=1}^{k} \int_{0}^{T} \int_{\mathbb{R}}\left(E\left[D_{s, x, j} \xi \mid \mathfrak{A}_{s}\right]-x \mathfrak{D}_{\eta_{j}} \xi(s)\right) \tilde{N}_{j}(d s, d x)$

Hence

$$
\begin{gathered}
E\left[\xi^{0} \Theta\right]=E \xi \cdot \sum_{j=1}^{k} E\left[\int_{0}^{T} \theta_{j}(s) d \eta_{j}(s)\right] \\
+\sum_{i, j=1}^{k} E\left[\int_{0}^{T} \theta_{i}(s) \sigma_{i} d W_{i}(s) \cdot \int_{0}^{T}\left(\frac{1}{\sigma_{j}} E\left[D_{s, j} \xi \mid \mathfrak{A}_{s}\right]-\mathfrak{D}_{\eta_{j}} \xi(s)\right) \sigma_{j} d W_{j}(s)\right] \\
+\sum_{i, j=1}^{k} E\left[\int_{0}^{T} \theta_{i}(s) \sigma_{i} d W_{i}(s) \cdot \int_{0}^{T} \int_{\mathbb{R}}\left(E\left[D_{s, x, j} \xi \mid \mathfrak{A}_{s}\right]-x \mathfrak{D}_{\eta_{j}} \xi(s)\right) \tilde{N}_{j}(d s, d x)\right] \\
+\sum_{i, j=1}^{k} E\left[\int_{0}^{T} \int_{\mathbb{R}} x \theta_{i}(s) \tilde{N}_{i}(d x, d s) \cdot \int_{0}^{T}\left(\frac{1}{\sigma_{j}} E\left[D_{s, j} \xi \mid \mathfrak{A}_{s}\right]-\mathfrak{D}_{\eta_{j}} \xi(s)\right) \sigma_{j} d W_{j}(s)\right] \\
+\sum_{i, j=1}^{k} E\left[\int_{0}^{T} \int_{\mathbb{R}} x \theta_{i}(s) \tilde{N}_{i}(d x, d s) \cdot \int_{0}^{T} \int_{\mathbb{R}}\left(E\left[D_{s, x, j} \xi \mid \mathfrak{A}_{s}\right]-x \mathfrak{D}_{\eta_{j}} \xi(s)\right) \tilde{N}_{j}(d s, d x)\right] .
\end{gathered}
$$

Thanks to the independence of $\eta_{1}, \ldots, \eta_{k}$ and the well-known isometry, see for instance p. 62 in Ikeda and Watanabe (1989), we obtain the following equality

$$
E\left[\xi^{0} \Theta\right]=\sum_{j=1}^{k} E\left[\int_{0}^{T} \theta_{j}(s) \cdot\left(\frac{1}{\sigma_{j}} E\left[D_{s, j} \xi \mid \mathfrak{A}_{s}\right]-\mathfrak{D}_{\eta_{j}} \xi(s)\right) \sigma_{j}^{2} d s\right]
$$




$$
+\sum_{j=1}^{k} E\left[\int_{0}^{T} \int_{\mathbb{R}} x \theta_{j}(s) \cdot\left(E\left[D_{s, x, j} \xi \mid \mathfrak{A}_{s}\right]-x \mathfrak{D}_{\eta_{j}} \xi(s)\right) \nu_{j}(d x) d s\right],
$$

which leads to

$$
\begin{gathered}
E\left[\xi^{0} \Theta\right]=\sum_{j=1}^{k} E\left[\int _ { 0 } ^ { T } \theta _ { j } ( s ) \cdot \left\{\sigma_{j} E\left[D_{s, j} \xi \mid \mathfrak{A}_{s}\right]\right.\right. \\
\left.\left.+\int_{\mathbb{R}} x E\left[D_{s, x, j} \xi \mid \mathfrak{A}_{s}\right] \nu_{j}(d x)-\mathfrak{D}_{\eta_{j}} \xi(s) \cdot\left(\sigma_{j}^{2}+\int_{\mathbb{R}} x^{2} \nu_{j}(d x)\right)\right\} d s\right]=0,
\end{gathered}
$$

for all $\Theta$ in $H(\eta)$, i.e. for all integrands $\theta=\left(\theta_{1}, \ldots, \theta_{k}\right)$. Therefore, the statement follows

$$
\varphi_{j}(s):=\mathfrak{D}_{\eta_{j}} \xi(s)=\frac{\sigma_{j} E\left[D_{s, j} \xi \mid \mathfrak{A}_{s}\right]+\int_{\mathbb{R}} x E\left[D_{s, x, j} \xi \mid \mathfrak{A}_{s}\right] \nu_{j}(d x)}{\sigma_{j}^{2}+\int_{\mathbb{R}} x^{2} \nu_{j}(d x)}
$$

It is worth noting that in the case of the pure jump Lévy martingale $\eta_{j}(t)=\int_{0}^{t} \int_{\mathbb{R}} x \tilde{N}_{j}(d s, d x)$, $0 \leq t \leq T,(j=1, \ldots, K)$ as integrator, representation (4.1) reduces to

$$
\varphi_{j}(s)=\mathfrak{D}_{\eta_{j}} \xi(s)=\frac{1}{\mu_{j}} \cdot \int_{\mathbb{R}} x E\left[D_{s, x, j} \xi \mid \mathfrak{A}_{s}\right] \nu_{j}(d x)
$$

with $\mu_{j}=\int_{\mathbb{R}} x^{2} \nu_{j}(d x)$ in $E \eta_{j}^{2}(t)=\mu_{j} t, 0 \leq t \leq T, j=1, \ldots, k$.

Moreover, for $\eta_{t}, 0 \leq t \leq T$, as the Wiener process with $\lambda_{j}=\sigma_{j}^{2}$ in $E \eta_{j}^{2}(t)=\mu_{j} t$, $0 \leq t \leq T$, and $k=K$ representation (4.1) reduces to the case of the Clark-HaussmannOcone formula - cf. (2.10).

\section{Examples.}

In the construction of hedging portfolios, it is important to obtain the processes explicitly. As mentioned in the introduction, the financial market will be complete if it is driven by a Wiener process and $k=K$. In this case the Clark-Haussmann-Ocone theorem leads to an explicit representation of the hedging portfolio in terms of a derivative. However, financial markets modeled by Lévy processes are in general not complete. Here the Clark-HaussmannOcone formula can be used to give the "closest" hedge to the desired claim under minimal risk, in the sense that the variance of the difference between the claim and the hedged wealth is minimal. As we will illustrate in the following examples, the Clark-Haussmann-Ocone formula is a theoretical characterization of the integrands in (2.2) that sometimes shortens the computation of the hedge substantially.

Example 5.a. Suppose the market is modeled by $S_{0}$ and $K$ independent Wiener processes $S=\eta=\left(\eta_{1}, \ldots, \eta_{K}\right)=\left(W_{1}, \ldots, W_{K}\right)(\mathrm{cf} .(1.1),(1.2))$ and we are allowed to trade only in $S_{0}$ and $\left(\eta_{1}, \ldots, \eta_{k}\right)$, where $k<K$ securities. Then the market is incomplete because of the given constraints.

For any $\xi \in \mathbb{D}_{1,2}$ the minimal variance hedge $\hat{\xi}$ in Theorem 4.1 is

$$
\varphi_{j}(s)=\mathfrak{D}_{W_{j}} \xi(s)=E\left[D_{s, j} \xi \mid \mathfrak{A}_{s}\right], \quad 0 \leq s \leq T,
$$


$(j=1, \ldots, k)$ Moreover, since we have

$$
\xi=E \xi+\sum_{j=1}^{K} \int_{0}^{T} E\left[D_{s, j} \xi \mid \mathfrak{A}_{s}\right] d W_{j}(s)
$$

- cf. (2.10), and also

$$
\xi=\xi^{0}+\hat{\xi}=\xi^{0}+\sum_{j=1}^{k} \int_{0}^{T} \varphi_{j}(s) d W_{j}(s),
$$

then the minimal risk can be given explicitly:

$$
\xi^{0}=\sum_{j=k+1}^{K} \int_{0}^{T} \varphi_{j}(s) d W_{j}(s) .
$$

Example 5.b. Let $K=1$ and $\eta(t)=W(t)+\int_{0}^{t} \int_{\mathbb{R}} x \tilde{N}(d s, d x), 0 \leq t \leq T$, so that $\mu=$ $1+\int_{\mathbb{R}} x^{2} \nu(d x)$. Suppose we want to hedge the claim

$$
\xi=\int_{0}^{T} \int_{\mathbb{R}} x^{p} \tilde{N}(d s, d x) \quad(p=0,1, \ldots) .
$$

Then $D_{s} \xi=0$ and $D_{s, x} \xi=x^{p}=E\left[D_{s, x} \xi \mid \mathfrak{A}_{s}\right]$. So, according to Theorem 4.1, the minimal hedging portfolio is a constant:

$$
\varphi(s)=\mathfrak{D}_{\eta} \xi(s)=\frac{1}{\mu} \int_{\mathbb{R}} x^{p+1} \nu(d x)=: \varphi, \quad 0 \leq s \leq T,
$$

and the minimal risk is given by:

$$
\xi^{0}=\int_{0}^{T} \int_{\mathbb{R}} x\left(x^{p-1}-\varphi\right) \tilde{N}(d s, d x)-\varphi W(T) .
$$

Example 5.c. Let $K=1$ and $\eta(t)=\int_{0}^{t} \int_{\mathbb{R}} x \tilde{N}(d s, d x), 0 \leq t \leq T$, so that $\mu=\int_{\mathbb{R}} x^{2} \nu(d x)$. Suppose $\xi=(\eta(T))^{2}$. Then, thanks to Lemma 9 in [L], we have

$$
D_{s, x} \xi=(\eta(T)+x)^{2}-(\eta(T))^{2}=x(2 \eta(T)+x), \quad 0 \leq s \leq T .
$$

So, the minimal variance portfolio is given by

$$
\varphi(s)=\mathfrak{D}_{\eta} \xi(s)=\frac{1}{\mu} \int x^{2}(2 \eta(s)+x) \nu(d x)=2 \eta(s)+\frac{1}{\mu} \int x^{3} \nu(d x), \quad 0 \leq s \leq T .
$$

\section{References.}

Aase, K., B. Øksendal, N. Privault, and J. Ubøe (2000): "White noise generalizations of the Clark-Haussmann-Ocone theorem with application to mathematical finance," Finance and Stochastics, 4, 465-496.

Bertoin, J. (1996): Lévy Processes, Cambridge: Cambridge University Press. 
Chan, T. (1999): "Pricing contingent claims on stocks driven by Lévy processes," Ann. Appl. Probab., 9, 504-528.

Di Nunno, G. (2001): "Stochastic integral representations, stochastic derivatives and minimal variance hedging," Preprint series in Pure Mathematics, University of Oslo, 19. To appear in Stochastics Stochastics Rep.

Di Nunno, G., and Yu. A. Rozanov (1999): "On stochastic integration and differentiation," Acta Appl. Math., 58, 231-235.

Föllmer, H., and M. Schweizer (1991): "Hedging of contingent claims under incomplete information," in Applied Stochastic Analysis: Stochastics Monogr. 5. New York: Gordon and Breach, 389-414.

Föllmer, H., and D. Sondermann (1986): "Hedging of non-redundant contingent claims," Contributions to mathematical economics. Amsterdam: North Holland, 205-223.

Ikeda, N., and S. Watanabe (1989): Stochastic Differential Equations and Diffusion Processes. Amsterdam: North-Holland.

Itô, K. (1956): "Spectral type of the shift transformation of differential processes with stationary increments," Trans. Am. Math. Soc., 81, 253-263.

Karatzas, I., D. Ocone and J. Li (1991): "An extension of Clark's formula," Stochastics Stochastics Rep., 37, 127-131.

Léon, J. A., J. L. Solé, F. Utzet and J. Vives (2002): "On Lévy processes, Malliavin calculus and market models with jumps," Finance Stochast., 6, 197-225.

Løkka, A. (2001): "Martingale representation of functionals of Lévy processes," Preprint series in Pure Mathematics, University of Oslo, 21.

Lamberton, D., and B. Lapeyre (1996): Introduction to Stochastic Calculus Applied to Finance. London: Chapman and Hall.

Malliavin, P. (1997): Stochastic Analysis. New York: Springer-Verlag.

Nualart, D. (1995): The Malliavin Calculus and Related Topics. New York: SpringerVerlag.

Nualart, D., and W. Schoutens (2000): "Chaotic and predictable representations for Lévy processes," Stochastic Process. Appl., 90, 109-122.

Nualart, D., and W. Schoutens (2001): "Backward stochastic differential equations and Feynman-Kac formula for Lévy processes, with applications in finance," Bernoulli, 7, 761-776.

Øksendal, B. (1996): An Introduction to Malliavin Calculus with Applications to Economics, Working Paper 3. Bergen: Norwegian School of Economics and Business Administration.

Protter, P. (1990): Stochastic Integration and Differential Equation. Belin: Springer.

Sato, K. (1999): Lévy Processes and Infinitely Divisible Distributions. Cambridge: Cambridge University Press.

Schweizer, M. (2001): "A guided tour through quadratic hedging approaches," in Option Pricing, Interest Rates and Risk Management, eds. E. Jouini et al.. Cambridge: Cambridge University Press, 538-574. 